\title{
DISERTACIONES
}

\section{EL EFECTO DEL AROMA EN LA INTENCIÓN DE COMPRA DE UN PAQUETE VACACIONAL}

\section{The Effect of Aroma on Intentions to Purchase Vacation Packages \\ O efeito do aroma na intenção de compra de um pacote turístico}

\section{Laura Pilar Cuatrecasas Clemente, Universitat Autònoma de Barcelona}

laucuatrecasas@gmail.com

Recibido: 13 de julio de 2016

Aceptado: 23 de enero de 2017

\section{RESUMEN}

Se estudia el efecto del aroma asociado a un destino turístico en la intención de compra de un paquete vacacional. Sesenta estudiantes universitarios ( $M=21,45$, DT $=1,50$ ) visualizaron un spot de un destino caribeño en una de las dos condiciones experimentales: sin aroma y con aroma ambiental asociado a un destino turístico. Tras el visionado, los participantes completaron un cuestionario que incluía escalas Likert. El análisis estadístico revela que el aroma asociado al destino turístico no tiene ningún efecto en la intención de comprar de un paquete vacacional, pero sí lo tiene el agrado por el video promocional. Estos resultados contribuyen al conocimiento de las propiedades del mercadeo experiencial aplicado a la industria del turismo.

Palabras clave: mercadeo experiencial, publicidad sensorial, publicidad olfativa, intención de compra, turismo. 


\section{DISERTACIONES}

Estudios de audiencias y recepción: audiencias minoritarias y nuevas mediaciones

ISSN: 1856-9536

Doi: http://dx.doi.org/10.12804/revistas.urosario.edu.co/disertaciones/v11i1

Volumen 11, Número 1 / Enero-junio 2018

Versión PDF para imprimir desde

http://revistas.urosario.edu.co/index.php/disertaciones

\section{ABSTRACT}

This study analyzes the effect of aromas associated with tourist destinations on the intention to buy a vacation package. Sixty university students $(M=21.45, S D=1.50)$ watched a video spot regarding a Caribbean destination in one of two experimental conditions: with no aroma, and with an environmental aroma associated with the tourist destination. After watching the spot, participants completed a questionnaire that included Likert scales. Statistical analysis reveals that the aroma associated with the tourist destination has no effect on the intention of buying a vacation package. On the other hand, experiencing pleasure while watching the promotional video does have an effect on this intention. These results contribute to the knowledge of the properties of experiential marketing applied to the tourism industry.

Keywords: Experiential marketing, sensory advertising, olfactory advertising, purchase intent, tourism.

\section{RESUMO}

Estuda-se o efeito do aroma associado a um destino turístico na intenção de compra de um pacote turístico. Sessenta estudantes universitários ( $M=21,45, D T=1,50)$ visualizaram um spot de destino caribenho em uma das duas condições experimentais: sem aroma e com aroma ambiental associado a um destino turístico. Depois de assistir, os participantes preencheram um questionário que incluía escalas Likert. A análise estatística revela que o aroma associado ao destino turístico não tem nenhum efeito na intenção de comprar um pacote turístico, mas sim o tem o agrado pelo vídeo promocional. Estes resultados contribuem ao conhecimento das propriedades do marketing experiencial aplicado à indústria do turismo.

Palavras-chave: marketing experiencial, publicidade sensorial, publicidade olfativa, intenção de compra, turismo.

\section{Introducción}

Desde hace algunos años, las empresas se enfrentan a un consumidor exigente que busca marcas adaptadas a los cambios e innovaciones del mercado, que se diferencien incrementalmente de su competencia. Precisamente por este motivo se produjo la evolución del mercadeo tradicional al experiencial, durante la década de los años ochenta. El mercadeo experiencial sitúa al consumidor en el centro del proceso y lo involucra emocionalmente en la compra mediante la creación de experiencias. Dentro de este mercadeo se encuentran las técnicas del mercadeo sensorial (Schmitt, 1999), que incluyen los sentidos exoreceptores de la vista, el oído, el olfato, el gusto y el tacto (Kohel, 2004). Como su nombre indica, el mercadeo sensorial busca ofrecer experiencias al cliente mediante estímulos sensoriales con el fin de obtener una respuesta en su conducta o comportamiento. En el campo del 


\section{DISERTACIONES}

ESTUDIOS

Estudios de audiencias y recepción: audiencias minoritarias y nuevas mediaciones

ISSN: 1856-9536

Doi: http://dx.doi.org/10.12804/revistas.urosario.edu.co/disertaciones/v11i1

Volumen 11, Número 1 / Enero-junio 2018

Versión PDF para imprimir desde

http://revistas.urosario.edu.co/index.php/disertaciones

mercadeo sensorial, algunos autores consideran que el sentido más influyente en los consumidores es el olfato, debido a su vinculación con el sistema límbico y consecuentemente con la vida emocional y la memoria de las personas (Cahill et al., 1995).

La investigación ha demostrado que existe una relación entre el sentido del olfato y el proceso de compra, por ser el aroma un elemento decisivo al momento de adquirir un producto o servicio (Canals, 2007; Gulas \& Bloch, 1995). Por ello no solo ha crecido el número de investigaciones que exploran la relación entre los aromas y la intención de compra, sino también sobre su relación con las estrategias de mercadeo. Generalmente, estas investigaciones observan los efectos del uso del aroma de cuatro formas distintas: a) como parte de las propiedades físicas del producto; b) como odotipo (logo olfativo de una marca); c) cuando se impregna en el papel que contiene la publicidad, y d) como aroma ambiental en espacios comerciales (Chebat \& Michon, 2003; Mitchell, 1994; Morrin \& Ratneshwar, 2000; 2003). Debido a que los resultados de las investigaciones, durante los últimos años tienden a encontrar correlaciones positivas entre la predisposición a la compra y el aroma, han proliferado los establecimientos que apuestan por usar un olor como estímulo para el comprador, ya sea a través de odotipos o de aromas ambientales.

Las investigaciones sobre el efecto del aroma en la intención de compra se han producido en variados campos (Díez, 2013), como la moda (Cox, 1967), los electrodomésticos (Airessentials, 2010a), los productos de cuero (Chadna, 2013), los casinos (Strozniak, 2013) y los hospitales (UAIRQ, 2012; Scentair, 2013). Ahora bien, dicha variedad no revela un conocimiento profundo del comportamiento de los clientes en cada ámbito específico, por lo que cabe más investigación aplicada (Díez, 2013), que justifica un trabajo como el presente. Existe además un sector industrial que tiene un vacío de conocimiento sobre el uso del olor en la intención de compra: el turismo. Aunque son muchos los autores que han explorado el efecto del mercadeo experiencial en otros ámbitos de esta industria, como en la intención de visitar o de volver a un lugar (Liao-ji, 2006; Pine \& Gilmore, 1998), se desconoce la existencia de investigaciones en este ámbito. En conjunto, los resultados disponibles muestran la influencia positiva del mercadeo experiencial como instrumento de persuasión para la selección de un destino turístico.

Con el fin de dar respuesta al vacío de conocimiento existente, esta investigación pretende observar el efecto del aroma en la intención de compra de un paquete vacacional ofertado en un spot publicitario. Los resultados de este trabajo serán, por una parte, de aplicación en el sector del turismo, un mercado que experimenta un crecimiento constante, aparte de grandes retos en sus mecanismos de altísima competencia. Así, se estima que para finales de 2016, el turismo en España creció de 4\% a 4,5\% respecto al año anterior (García, 2016). Por ello, el estudio de la eficacia de distintas técnicas de mercadeo experiencial y sensorial puede ofrecer conocimiento útil para atraer viajeros a los destinos turísticos sin la necesidad de contar con la experiencia de quienes hayan visitado con anterioridad el lugar. Esto, en particular, supondría una ayuda para aquellos destinos turísticos que no poseen recursos para mostrar todo su potencial. Asimismo, una investigación como esta podría enriquecer el conocimiento sobre los mecanismos que potencian la eficacia de los mensajes persuasivos y por tanto aportar información útil para la publicidad. Además, los resultados de este trabajo permitirían informar sobre la relación entre los estímulos perceptivos comerciales de naturaleza audiovisual y olfativa. Finalmente, este estudio se propone como una aportación de interés para la gerencia de los negocios que se sostienen a partir de la relación comercial con clientes en puntos de venta de distinta naturaleza. 


\section{DISERTACIONES}

ESTUDIOS

Estudios de audiencias y recepción: audiencias minoritarias y nuevas mediaciones

ISSN: 1856-9536

Doi: http://dx.doi.org/10.12804/revistas.urosario.edu.co/disertaciones/v11i1

Volumen 11, Número 1 / Enero-junio 2018

Versión PDF para imprimir desde

http://revistas.urosario.edu.co/index.php/disertaciones

\section{Mercadeo experiencial}

El mercadeo experiencial como concepto se origina en las obras Experiential Marketing (Schmitt, 1999) y Customer Experience Management (Schmitt, 2003). En ellas, Schmitt reta al mercadeo tradicional cuando propone un nuevo enfoque centrado en el consumidor, que busca crear un vínculo emocional con el bien o servicio derivado de la experiencia de adquirirlo y no con los beneficios funcionales o racionales que mueven al consumidor a comprarlo.

Distintos autores han aportado definiciones propias del mercadeo experiencial, en buena parte debido a las áreas del conocimiento desde las que se le aproxima, como la psicología, la economía o el mercadeo (Carú \& Cova, 2003). Sin embargo, se observan coincidencia en algunos aspectos de esas definiciones. Por un lado, en ellas aparece el término interacción (Boyd \& Levy, 1963; Hoch 2002; IXMA, 2005; Kotler, 1999; Schmitt, 1999) referido a un contacto entre algún elemento del producto o servicio y el consumidor. La interacción en el mercadeo experiencial tiene que ver con la existencia de una comunicación bilateral entre la marca y el cliente. Otras definiciones, por su parte, coinciden en implicar la idea de recuerdo (Oliver, 1997; Pine \& Gilmore, 1999), específicamente en el hecho de que el mercadeo experiencial se refiere a una acción o actividad que queda establecida en la mente a largo plazo. Ahora bien, existe un aspecto en el que coinciden todas las definiciones: la experiencia del consumidor.

Desde la perspectiva que ocupa esta investigación en el mercadeo existen distintas definiciones de experiencia (Brakus, Schmitt \& Zarantonello, 2009; Holbrook, 2000; Lenderman, 2008; Shaw \& Ivens, 2005). Holbrook y Hirschman (1982) fueron de los primeros en aproximarse a su definición. Para ellos, se trata de una experiencia vinculada al comportamiento del consumidor, que se convierte en un acontecimiento personal con significado emocional importante. De acuerdo con los autores, dicha experiencia debe suponer la interacción con un estímulo provocado por el consumo de un producto o servicio. Otro autor, Schmitt (1999), acuña el término experiencia del cliente (customer experience) para definir las interacciones entre un consumidor y un producto, compañía o elemento de una empresa, que ocasionan reacciones positivas en el sujeto. El mismo autor define los módulos estratégicos experienciales (strategic experiential modules, SEM), que conforman la base estratégica del mercadeo experiencial: las sensaciones, los sentimientos, los pensamientos, las actuaciones y las relaciones.

Las primeras investigaciones sobre mercadeo experiencial datan de la década de los años ochenta, pero es a partir de finales de la siguiente década cuando se empieza a aplicar en diferentes áreas de conocimiento. Así, Holbrook y Hirschman (1982) en la obra The Experiential Aspects of Consumption: Consumer Fantasies, Feelings and Fun hablaron, por primera vez, de las emociones como factor clave en el comportamiento de los consumidores, donde mostraron una visión experimental centrada en la naturaleza simbólica. Además, los autores introdujeron el concepto de hedonismo en el proceso de compra en oposición a la idea vigente de racionalidad. El consumo hedónico adopta una visión más subjetiva de los productos y servicios, basada en las experiencias de consumo y no en la funcionalidad de ellos (Holbrook \& Hirschman, 1982). A partir de los argumentos de estos autores se inician nuevas investigaciones, como las de Csikszentmihalyi (1990) y Carlson (1997) que contemplan aspectos emocionales del consumo. Para estos autores, la experiencia consiste en un flujo de sentimientos y pensamientos conscientes sobre las dimensiones de la experiencia. También destaca la aportación de Pine y Gilmore (1998) que añade una perspectiva económica al acuñar el concepto de economía de la experiencia (experience economy): 


\section{DISERTACIONES}

ESTUDIOS

Estudios de audiencias y recepción: audiencias minoritarias y nuevas mediaciones

ISSN: 1856-9536

Doi: http://dx.doi.org/10.12804/revistas.urosario.edu.co/disertaciones/v11i1

Volumen 11, Número 1 / Enero-junio 2018

Versión PDF para imprimir desde

http://revistas.urosario.edu.co/index.php/disertaciones

una nueva estructura de consumo en que las empresas que quieren competir en un mercado con garantías deben incluir experiencias como elemento diferenciador de sus productos o servicios.

En la bibliografía de mercadeo experiencial son relevantes, además de las aportaciones citadas, la de otros autores que en conjunto han aplicado sus teorías a diferentes ámbitos y problemas (Brakus, Schmitt \& ZarantoneIlo, 2009; Hui-Yi y Hung-Yuan, 2010; Lenderman \& Sánchez, 2008; Schmitt \& Zarantonello, 2013). Concerniente con el turismo, existen diversas investigaciones que estudian la experiencia turística desde diferentes perspectivas y variables, aunque la mayoría estudian la experiencia una vez el turista ha visitado el destino (Back, 2015; Craig, Smith, \& French, 1994; Liao-ji, 2006; Parasuraman, 1985; Smith, 2005; Sotomayor, 2012; Zeithamal \& Berry, 1998; Walls et al., 2011; Wang, 1999).

Una contribución relevante para este trabajo es la de Oh et al. (2007), quienes analizan la importancia de los cuatro tipos de experiencia de Pine y Gilmore (1998) en el turismo bed\&breakfast. Sus resultados sugieren que los factores que predicen las motivaciones para escoger un destino turístico están vinculados a la experiencia percibida del destino. Según la investigación, los clientes buscan cuatro tipos de experiencias: 1) novedosa o relajante; 2) seguimiento de tendencias; 3) utilidad física, y 4) accesibilidad y socialización. Por su parte, Uriely (2005) desarrolla las teorías posmodernistas sobre la experiencia turística y mediante ellas afirma que un turista puede vivir experiencias turísticas sin tener que viajar, gracias a la posibilidad de crear una simulación de ambiente. Precisamente en esta línea, Jiménez y Campo (2016) realizan una investigación en la Feria Internacional de Turismo de España (FITUR), que demuestra la existencia de una relación positiva entre la experiencia vivida en el stand de un destino turístico y la intención de visitar ese lugar. Otras aportaciones dentro del contexto del turismo, vinculadas a la experiencia (Aertsen, 2011; Agapito, 2012; Gong \& Tung, 2016; Hudson \& Ritchie, 2008) afirman la correlación entre la inducción de experiencias en los consumidores y la valoración positiva de los bienes o servicios turísticos.

\section{Mercadeo sensorial}

El mercadeo sensorial es considerado parte del experiencial (Schmitt, 1999). Los trabajos sobre este tipo de mercadeo otorgan el nacimiento del concepto a Schmitt y Simonson (1998), quienes, a su vez, definen su objetivo: llegar a las personas a través del hemisferio derecho del cerebro, que es el que maneja las emociones, en lugar de mediante el izquierdo, que es el que utiliza la razón y la lógica. Otros autores afirman que el mercadeo sensorial se basa en crear experiencias mediante la introducción de estímulos sensoriales controlables y modificables en un entorno, con el fin de obtener una reacción o respuesta determinada del consumidor provocada de forma irracional y emocional por dichos estímulos (Filser, 2003; Manzano et al., 2010; Rieunier 2004). Digout (2007) y Herrera (2012) afirman que el mercadeo sensorial se basa en crear experiencias memorables que el consumidor pueda reconocer; para Wright et al. (2006) las grandes compañías se esfuerzan en tener éxito dando poder a los consumidores en sus actividades de mercadeo, en vez de manipularles. Según estos autores, el mercadeo sensorial no implica manipulación no consciente. Sin embargo, en él, los impulsos juegan un papel mucho más fuerte y predominante que la razón, por lo que los consumidores se dejan llevar por sus emociones y no por la racionalidad (Koehl, 2004). De acuerdo a Schmitt (1999), los tres objetivos del mercadeo sensorial para una empresa son: diferenciarse de la competencia, motivar a los consumidores y dar valor a los clientes. Para alcanzar estos tres objetivos y lograr impacto sensorial, el autor establece un modelo E-P-c (estímulo-proceso-consecuencia) de 


\section{DISERTACIONES}

ESTUDIOS

Estudios de audiencias y recepción: audiencias minoritarias y nuevas mediaciones

ISSN: 1856-9536

Doi: http://dx.doi.org/10.12804/revistas.urosario.edu.co/disertaciones/v11i1

Volumen 11, Número 1 / Enero-junio 2018

Versión PDF para imprimir desde

http://revistas.urosario.edu.co/index.php/disertaciones

sensaciones: un estímulo particular invita a atender a una información sensorial. El estímulo es conducido hasta el consumidor siguiendo procesos sensoriales (auditivos, visuales, olfativos o táctiles). La consecuencia del proceso es la comprensión y recuerdo del estímulo por el consumidor.

\section{Los sentidos exoreceptores}

Según Koehl (2004), los cinco sentidos que el mercadeo sensorial incluye son la vista, el oído, el olfato, el gusto y el tacto. Llegados a ese punto, es preciso considerar que la investigación científica ha escapado de esta definición simplista, basada en textos antiguos, sobre los sentidos humanos que sostiene la existencia de los solo 5 sentidos. En la actualidad, se sabe que el proceso de recepción sensorial es más complejo. No obstante, debido a que buena parte de la literatura relacionada refiere la clásica definición, en este estudio los referimos acorde a los autores citados.

Estanyol (2014) afirma que las personas recordamos el 35\% de lo que olemos, el 15\% de lo que saboreamos, el $5 \%$ de lo que vemos, el $2 \%$ de lo que oímos y el $1 \%$ de lo que tocamos. La vista es uno de los sentidos más utilizados en el mercadeo, ya que la variedad de estímulos que se pueden utilizar es muy amplia. Según Schmitt y Simonson (1998), cualquier percepción se inicia a través de los ojos, además en el ámbito del mercadeo de la estética los elementos que más destacan son los visuales. Para Hultén et al. (2009), la mayoría de la decisiones cotidianas de las personas están basadas en impresiones visuales. Por su parte, el sentido del oído hace referencia a todo lo relacionado con los sonidos. Los sonidos son importantes como fondo y como creadores de identidad de marca (Schmitt \& Simonson, 1998). Aun así, cuando nos referimos a los procesos de compra, muchos investigadores señalan a la música como componente principal e imprescindible (Areni \& Kim, 1993; Milliman, 1982; 1986; Turley \& Milliman, 2000). El sentido del gusto es uno de los menos estudiados en el mercadeo sensorial. Se refiere a los estímulos a través de la lengua y las papilas gustativas. Hultén et al. (2009) ponen espacial énfasis en destacar la estrecha relación entre el sentido del gusto y el sentido del olfato. Ambos forman los sentidos humanos químicos, es decir, permiten reconocer elementos químicos en el ambiente. De hecho, los autores consideran que el olfato es esencial para la habilidad de distinguir sabores. Finalmente, el tacto, que es el menos estudiado de los cinco (Peck \& Childers, 2007), es el sentido humano más antiguo y primitivo. En realidad, es el primer sentido que se experimenta (desde el útero) y el último que se pierde (Raisamo et al., 2007). Estas autoras, sin embargo, y en coherencia con las investigaciones en neuropsicología más recientes, consideran que limitar los sentidos a cinco significa ignorar la complejidad sensorial real de los seres humanos. No obstante, debido a que es desde esta perspectiva, fundamentada en los conocimientos de la antigua Grecia, desde la cual se aborda el estudio del mercadeo sensorial, no se da cuenta de los conocimientos manejados en la actualidad dentro del área.

De los cinco sentidos, el sentido del olfato es el más primitivo, debido a su estrecha relación con el sistema límbico, la estructura cerebral que se ocupa de las emociones y la construcción de la memoria (Arboleda, 2015; Cahill, Markowitsch, McGaugh \& Babinsky, 1995; Ward et al. 2003). Para Schmitt y Simonson (1999), el sentido del olfato también es el más poderoso, debido a la omnipresencia de los aromas en el ambiente. Según Navalles (2011), las personas cuentan con numerosos aromas almacenados en la memoria que responden a sus preferencias de consumo, cultura, sociedad, procedencia y estatus social. Aun así, en el mercadeo olfativo, es imprescindible que el sujeto haya estado expuesto a una experiencia para que la pueda asociar después con un aroma. De hecho, las personas tendemos a relacionar los aromas con percepciones mentales. Por ejemplo, vinculamos el invierno al 


\section{DISERTACIONES}

ESTUDIOS

Estudios de audiencias y recepción: audiencias minoritarias y nuevas mediaciones

ISSN: 1856-9536

Doi: http://dx.doi.org/10.12804/revistas.urosario.edu.co/disertaciones/v11i1

Volumen 11, Número 1 / Enero-junio 2018

Versión PDF para imprimir desde

http://revistas.urosario.edu.co/index.php/disertaciones

pino o la canela (Schmitt \& Simonson, 1999). Sobre el mismo tema, Rieunier (2004) afirma que los aromas tienen la capacidad de evocar recuerdos y provocar emociones, ya sean positivas o negativas. Además, Cain (1979) sostiene que la facilidad para reconocer un aroma es mayor cuando el sujeto asocia el contexto a un momento concreto. Hay estudios que identifican la importancia de un aroma para generar respuestas positivas en los clientes (Chebat \& Michon, 2013; Romera, 2014).

\section{Olfato e intención de compra}

La intención es definida como la necesidad de un individuo para alcanzar una determinada condición en el futuro (Williams, 1972). Arrebola (1983) sostiene que un proceso en la decisión de compra es la decisión de gastar dinero o no, para obtener una satisfacción material o inmaterial. Cuando un consumidor compra un producto inmaterial, sabe que existen riesgos de que finalmente no se cumplan sus expectativas y, además, su grado de renuncia y miedo es proporcional a la cantidad de dinero que va a invertir en relación con sus recursos totales (Wahab, Crampón \& Rothfield, 1976).

El olfato es el sentido con un mayor grado de percepción y, como se ha indicado anteriormente, puede influir en emociones, pensamientos y acciones. Canals (2007) defiende que la emoción producida por un olor puede ser el factor decisivo al momento de decidir realizar una compra. Por su parte, Morrin y Ratneshwar (2000) refuerzan esta idea al añadir que el nivel de aceptación y agrado que se percibe de un aroma en un lugar concreto puede mejorar tanto el proceso de compra, como la evaluación que se le hace a ciertos productos que, a simple vista, no resultan atractivos. Otros autores contribuyen a demostrar la existencia de una relación positiva entre el aroma y la intención de compra (Bone \& Jantrania, 1992; Gulas \& Bloch, 1995; Krishna, 2012; Mitchell et al., 1995; Olahut \& Plaias, 2013; Spangenberg et al., 1996). Un estudio fundamental es el de Cox (1967), que reporta que las clientas de un establecimiento de moda íntima preferían unas medias con aroma a naranja a otras sin aromas, debido a que las compradoras percibían un aroma agradable. Este era, a su vez, asociado por las clientas al producto por la frescura que el olor cítrico transmitía.

\section{El turismo}

La imagen de un destino turístico es un constructo multidimensional que consiste en la combinación de factores emocionales y racionales (Frías, Rodríguez \& Castañeda, 2008). Asimismo, la imagen incide en el comportamiento de los turistas, desde sus percepciones mentales sobre el lugar hasta el proceso de decisión de visitarlo (Prayag, 2009).

Arrebola (1983) distingue entre la decisión de compra rutinaria y la decisión de compra por un impulso atrayente. Según el autor, la primera se da en el turismo siempre y cuando la información se haya obtenido a través de mercadeo turístico: el turista puede actuar por sugestión ya que cuenta con información sobre el lugar, recopilada a través del tiempo y obtenida de diferentes fuentes. Por el contrario, la segunda sería aquella en que el turista recibe estímulos externos a él que le provocan la necesidad de visitar el destino. Wahab, Crampón y Rothfield (1976) aíslan los aspectos que los turistas tienen en cuenta antes de decidir invertir en un viaje. Todos ellos, sin embargo, coinciden en reflejar la importancia que la economía tiene para los turistas. De hecho, los turistas, sobre todo, tienen en cuenta su estado financiero a la hora de decidir viajar a un destino turístico.

\section{6}




\section{DISERTACIONES}

ESTUDIOS

\section{Turismo y mercadeo sensorial}

El sector del turismo se enfrenta a un alto nivel de competencia que provoca que las empresas tengan que centrar sus esfuerzos en ofrecer experiencias multisensoriales a sus clientes (Ritchie, 2010). Una experiencia turística engloba todo lo que sucede fuera de la rutina cotidiana de las personas (Walls et al., 2011). Según Braidot (2010, citado por Sotomayor, 2012)), en el mercadeo turístico, un destino se posicionará exitosamente si logra integrar de forma adecuada los diferentes estímulos sensoriales para provocar en el turista sentimientos y emociones favorables. Sin embargo, es imprescindible controlar la veracidad y la calidad de la información dada a los posibles visitantes de un destino, ya que a partir de ella se generan sus expectativas. Por ello, se aconseja evitar que haya una enorme diferencia entre lo que el turista espera encontrar y lo que encontrará realmente (Braidot, 2010). También resulta esencial para una empresa saber elegir los estímulos sensoriales adecuados para incidir en la conducta del consumidor, en el momento en que está decidiendo qué destino prefiere. En este ámbito, Sotomayor (2012) realiza un estudio que analiza cómo afecta cada sentido la visita a un destino turístico. Según el autor, la experiencia turística es una realidad construida socialmente e influenciada por la experiencia, que el cerebro conduce. Específicamente respecto al olfato, Sotomayor considera que este sentido es el responsable de las tres cuartas partes de las emociones generadas por el turista. Además, afecta positiva o negativamente a distintos órganos cerebrales: los olores agradables asociados a un destino estimulan los lóbulos frontales, mientras que los desagradables estimulan la amígdala y la corteza del lóbulo temporal. Por ello, los turistas construyen imágenes mentales del destino que se interpretan según dónde se sitúa su atención. A pesar, de ello, aún hoy día se carece de estudios empíricos que demuestren consistentemente la relación entre la intención de compra de un paquete vacacional y los estímulos olfativos. Este estudio pretende contribuir en esta área. Por todo lo anterior, la presente investigación supone que:

H1: La percepción de un aroma asociado a la visualización de un destino turístico incrementará la intención de comprar un paquete turístico.

De ello, a su vez, se deduce que la presente investigación se pregunta, específicamente:

PI1: ¿En qué medida un aroma asociado a un destino caribeño incita a la compra de un paquete vacacional a esa destinación, cuando es ofertado en un vídeo promocional?

En consecuencia, este estudio también explora la relación entre la percepción audiovisual y la olfativa en el mercadeo turístico.

\section{Método}

Para dar respuesta a la pregunta de investigación y confirmar la hipótesis se condujo un estudio experimental básico en el que se manipuló una variable independiente (actuación de aroma) y se observó su efecto en la variable dependiente (intención de compra).

\section{Participantes}

En el estudio tomaron parte 60 estudiantes universitarios de la Facultad de Ciencias de la Comunicación de la Universidad Autónoma de Barcelona. Los participantes eran de ambos sexos ( $54,1 \%$ mujeres y $44,3 \%$ hombres). La edad 


\section{DISERTACIONES}

ESTUDIOS

Estudios de audiencias y recepción: audiencias minoritarias y nuevas mediaciones

ISSN: 1856-9536

Doi: http://dx.doi.org/10.12804/revistas.urosario.edu.co/disertaciones/v11i1

Volumen 11, Número 1 / Enero-junio 2018

Versión PDF para imprimir desde

http://revistas.urosario.edu.co/index.php/disertaciones

promedio fue de 21,45 años (DT=1,50, $\mathrm{Rg}_{\text {Edad }}=19-26$ años). Todos los sujetos participaron de forma voluntaria y totalmente gratuita.

\section{Materiales}

Cuestionarios. Fueron construidos a partir de la escala de intención de compra de Dodds, Monroe y Grewal (1991), utilizada también por Grewal, Monroe y Krishnan (1998). Se complementó dicha escala con la de Baker y Crompton (2000) de atributos percibidos en el destino turístico, para lograr una medición lo más ajustada posible. Todos los ítems fueron traducidos del inglés al castellano y adaptados a los propósitos del estudio (ver anexo 1). La primera escala (INT) estaba formada por cinco ítems y contenía siete puntos, donde 1 correspondía a "muy baja" o "totalmente en desacuerdo" y 7, a "muy alta" o "totalmente de acuerdo". La segunda escala (веH) contenía seis ítems y nueve puntos, donde 1 era "nada probable" y 9, "muy probable".

Video promocional. Se seleccionó un video promocional sobre un destino turístico paradisíaco: República Dominicana. Fue elegido ese spot por las siguientes razones: contenía todas las imágenes idílicas y características de un paraíso turístico; de la misma forma, la música era coherente con estas. También, contaba con una duración de cinco minutos con cincuenta y cinco segundos, duración que permitía la inmersión de los participantes para su posterior valoración y evaluación. Finalmente, se escogió este vídeo por ser un destino popular entre los estudiantes y una opción frecuente para realizar el viaje de fin de estudios universitarios. El video fue modificado en la parte final: se añadieron elementos hipotéticos e inventados que daban información sobre el tipo de paquete turístico que se anunciaba: la categoría del hotel (cinco estrellas), la duración del viaje (7 días), el precio (1500€) y lo que la oferta incluía (vuelo+hotel).

Aroma. Se adquirió un ambientador con aroma a coco que conformó el estímulo olfativo para la segunda condición experimental. Tras buscar y comparar diferentes perfumes y ambientadores, se optó por un ambientador con un olor frutal concentrado, con el fin de que el aroma a coco fuese intenso y duradero. El olor a coco es asociado, generalmente, a destinos de playa y en particular a playas paradisíacas caribeñas.

\section{Procedimiento}

Los participantes fueron asignados aleatoria y equitativamente a cada condición experimental. La sala en que se llevó a cabo la prueba estaba aislada acústicamente y fue la misma para ambas condiciones experimentales: sin aroma y con aroma. En ambas se llevó a cabo el mismo proceso. Cada grupo de participantes entraba en la sala, el investigador les daba las indicaciones: en primer lugar debían visionar un spot de unos cinco minutos y, posteriormente, responder un cuestionario sobre sus impresiones acerca de él. En ningún momento se mencionó el objeto de estudio, con el fin de evitar cualquier condicionamiento. El investigador permaneció en todo momento en la sala para asistir, si era necesario, a los participantes. El anuncio fue mostrado a través de una pantalla de 15'. Antes de proceder al experimento se comprobó la correcta visualización y escucha desde todos los puntos del espacio. El primer grupo, sujeto a la primera condición experimental (sin aroma) entró a la sala en subgrupos de 4 a 10 personas. Los participantes visualizaron el video promocional y respondieron el cuestionario. Una vez los sujetos de la primera condición experimental finalizaron, se ventiló un rato la sala para eliminar cualquier aroma que pudiera interferir en el que se aplicaría para la segunda condición experimental (con aroma). Después, se roció el mismo 


\section{DISERTACIONES}

ESTUDIOS

espacio con la ayuda de un ambientador con aroma a coco. Se esparció abundante y generosamente el aroma por el suelo, mesas y paredes de la sala. Las dimensiones reducidas de esta facilitaron que el olor se concentrase y no dejase de estar presente durante toda la segunda parte de la investigación. Considérese, además, que los mismos papeles utilizados para realizar el cuestionario absorbieron el olor y eran otro elemento de difusión de este. Cabe destacar que en la segunda condición experimental se contó con la ayuda de personas ajenas a la investigación que comprobaron la presencia notable del aroma en el ambiente. No obstante, el estudio también tuvo la ayuda de una persona que actuaba de cómplice y que, haciéndose pasar por participante, aprovechaba para pulverizar más ambientador durante la presencia de los demás sujetos, de forma oculta. En la segunda parte del estudio, los sujetos también entraron a la sala en subgrupos y, de la misma manera que el primer grupo, visualizaron el spot y completaron el cuestionario posteriormente.

\section{Análisis de resultados}

Para el análisis y evaluación de resultados, se elaboró una matriz de datos por variables en formato IBM SPSS Statistics versión 22. Una vez generada la matriz, se realizó primeramente un análisis descriptivo para verificar la normalidad de estos: se calculó la F de Levene que permitió comprobar la homocedasticidad (homogeneidad de varianzas). Posteriormente, se emplearon pruebas de estadística inferencial para contrastar las hipótesis. Se aplicó una prueba t-Student para análisis de muestras independientes. Tal y como afirma Igartua (2006), la t-Student es la prueba paramétrica más popular para establecer una comparativa entre dos condiciones experimentales y se utiliza para comparar las medias de dos grupos en una variable criterio. Asimismo, en el caso de las variables categóricas ordinales se usó la prueba de U Mann-Witney.

\section{Resultados}

\section{Análisis descriptivo}

Primero, se realizó un análisis factorial exploratorio de componentes principales y rotación Oblimin con Kaiser con el objetivo de determinar cuál era la relación que existía entre las dos escalas y sus ítems. Para ello, se añadieron a la solución explorada todos los ítems de las dos escalas utilizadas. Los resultados obtenidos mostraron una estructura que diferenciaba claramente dos factores que explicaban, en conjunto, $61,9 \%$ de la varianza. El primer factor estaba formado por los ítems: "La probabilidad de que compre este producto es" (INT1), "Si comprara este producto consideraría comprarlo al precio que se muestra" (INT2), "Con el precio que se muestra consideraría comprarlo" (INT3), "La probabilidad de que considere comprar este paquete turístico es" (INT4), "Mi disposición a comprar este paquete turístico es" (INT5), "Compraría el paquete turístico aunque subiera de precio" (BEH1) y "Pagaría un precio más alto que para un paquete turístico similar" (BEH2). Tras estudiar la estructura de los anteriores ítems y en atención a sus componentes se pasó a llamarles "Intención de pagar", ya que todos ellos hacían referencia a cuestiones económicas y financieras en la decisión de compra. El segundo factor estaba formado por los ítems siguientes: "Diría cosas positivas sobre el destino turístico a otras personas" (BEH3), "Me cansaría de visitar el destino turístico cada año" (BEH4) y "Recomendaría el paquete turístico a amigos y familiares" (BEH5). Este factor se llamó "Intención de ir", debido a que tras observar los ítems se vio que consistían en elementos más 


\section{DISERTACIONES}

ESTUDIOS

comportamentales en la decisión de compra. Aunque la varianza detallada anteriormente (61,9\%) explica que las variaciones en la intención de compra de los sujetos experimentales se deben a los factores mencionados anteriormente, cabe considerar, sin embargo, que existen otros factores desconocidos $(38,1 \%)$ que también podrían afectar las respuestas de los participantes.

\section{Análisis inferencial}

Se realizó la prueba U Mann-Whitney que arrojó que no había diferencias estadísticas en función de la condición experimental (con o sin aroma) ni en la "Intención de pagar" ni en la "Intención de ir". También se exploró si la condición experimental afectaba el agrado por el vídeo. Tampoco se encontraron diferencias estadísticas.

Para explorar con más profundidad los aspectos particulares involucrados, se decidió analizar si la condición experimental influía en las respuestas obtenidas para cada ítem. Se aplicó el test t-Student para muestras independientes a cada uno y se observó que la condición experimental solamente tendía a afectar de forma estadísticamente significativa al ítem "La probabilidad de que compre este producto es" (INT1) ( $F=, 182, p=, 671, t=-3,041$, $g l=58, p=, 004)$. En la condición experimental 1 , sin aroma, los sujetos atribuyeron superiores índices al mismo ítem ( $M=4,63, D T=1,73)$ que en la condición experimental 2, con aroma ( $M=3,50, D T=2,34)$. En consecuencia, la condición experimental afecta de forma estadísticamente significativa la probabilidad que los participantes pensaran en pagar otro precio, y no la decisión de compra. Para esta muestra, la percepción del aroma incrementa la probabilidad percibida de que los participantes compren el producto turístico.

A continuación se exploró si el agrado por el vídeo había afectado a los dos factores identificados ("intención de compra" e "intención de ir"). Se realizó otra t-Student para muestras independientes y se obtuvo que, en conjunto, el video sí influye en la intención de pagar $(t=2,30, p=, 025)$, pero no en la de intención de ir. Las personas a quienes les gustó más el video, atribuyeron más intención de pagar ( $M=3,59, \mathrm{DT}=1,28)$ que aquellos a quienes no les gustó el video ( $M=2,26, \mathrm{DT}=1,86)$.

Para profundizar, igual que anteriormente, se analizaron los resultados de cada uno de los ítems. Así, se observó que el agrado por el spot influía en la probabilidad de compra ( $t=3,42, g l=6,29, p=, 013)$. Los sujetos a quienes les gustó el video atribuyeron mayores promedios de probabilidad de compra ( $M=4,20, D T=1,68)$ que a los que no les gustó el video ( $M=1,83, \mathrm{DT}=1,60)$.

Además, se encontró un efecto en quienes pagarían un precio más alto por un paquete turístico similar ( $t$ $=2,30, \mathrm{gl}=58, \mathrm{p}=, 025$ ). Los que manifestaron que sí les había gustado el video otorgaron una mayor media ( $\mathrm{M}=3,04$ $\mathrm{DT}=1,68)$ que aquellos que dijeron que no les había gustado ( $M=1,50 \mathrm{DT}=5,48)$.

Por último, se observó una influencia en el ítem "Si el paquete turístico no existiera, no me importaría, iría a un destino similar" (BEH6) ( $\mathrm{t}=2,30, \mathrm{gl}=58, \mathrm{p}=, 025)$. Las personas que manifestaron agrado por el video dijeron que no les importaría ir a un destino similar si no estuviera el paquete disponible en mayor medida $(M=6, D T=2,21)$ que las personas a quienes no les había gustado el video ( $M=4,67$ y $D T=3,20)$.

Por tanto, en líneas generales, se observó que el aroma como condición experimental no afecta la decisión e intención de compra. Sin embargo, lo que sí afecta la intención de compra es el agrado por el spot. 


\section{DISERTACIONES}

ESTUDIOS

Estudios de audiencias y recepción: audiencias minoritarias y nuevas mediaciones

ISSN: 1856-9536

Doi: http://dx.doi.org/10.12804/revistas.urosario.edu.co/disertaciones/v11i1

Volumen 11, Número 1 / Enero-junio 2018

Versión PDF para imprimir desde

http://revistas.urosario.edu.co/index.php/disertaciones

\section{Contraste de hipótesis}

Con los resultados obtenidos, se ve que la hipótesis formulada: "El aroma asociado a un destino turístico caribeño incrementará la intención de comprar un paquete turístico" queda casi totalmente refutada por el estudio experimental.

\section{Discusión}

La principal contribución de esta investigación es que ofrece información académica sobre el impacto del aroma en la intención de compra de un paquete vacacional. Asimismo, el estudio avanza en la compresión de la relación entre la percepción audiovisual y la olfativa en experiencias de mercadeo olfativo.

Este estudio tenía por objetivo comprobar si, tal y como dicen las teorías del mercadeo experiencial y más concretamente de mercadeo sensorial, el aroma asociado a un lugar es un factor que podría incidir en la intención de compra de un paquete turístico o en la intención de visitar un destino turístico. En este sentido, las conclusiones de este estudio contribuyen al conocimiento del objeto de estudio, a la vez que realiza aportaciones industriales al sector del turismo, la publicidad y el mercadeo. Sus resultados pueden contribuir al sector del turismo porque proporcionan información que puede resultar útil para las compañías dedicadas a la venta de paquetes turísticos. Asimismo, esta investigación contribuye al sector de la publicidad porque observa la relación entre un spot publicitario y su efecto en interacción con estrategias de mercadeo o modalidades de percepción sensorial. Además, el estudio se suma al largo cuerpo de investigación de otros autores en mercadeo experiencial (Schmitt, 1999; Holbrook \& Hirschman, 1982) que estudian el mercadeo olfativo (Schmitt \& Simonson, 1998; Koehl, 2004; Hultén et al., 2009), añadiendo conocimiento e informando a la teoría. Aun así, los resultados contradicen algunas afirmaciones de estudios en estos ámbitos, tal y como se detalla a continuación.

La primera conclusión extraída de este estudio es que el aroma asociado a un destino promocional turístico no tiene efecto en la intención de compra del mismo. Así, se observa que estos resultados obtenidos contradicen la afirmación de Canals (2007), quien afirma que los efectos de un aroma pueden ser cruciales para la decisión de compra de un producto o servicio. Los resultados de nuestra prueba experimental indican que en el caso de un destino turístico caribeño, y para esta muestra, no se producen los efectos predichos en la decisión de compra. Futuros estudios deberían comprobar si estos resultados se confirman tomando en consideración la intención real de compra de un paquete vacacional. Pudiera suceder que la inminencia de esta decisión sea un aspecto relevante. Asimismo, otros estudios deberían contrastar estos resultados con otros olores (inclusive desagradables) y otros destinos turísticos. También, deberían controlar la percepción del olor por parte de los participantes.

Por otra parte, queda abierta la opción de explorar en investigaciones futuras si los cuatro sentidos restantes (vista, oído, gusto y tacto) tienen algún efecto en la intención de adquirir un paquete turístico. De la misma forma, se podría profundizar investigando en otros sectores o contextos industriales diferenciados al de este trabajo. Se podría probar modificando la variable dependiente (vídeo promocional), ya sea mediante el cambio de duración de esta o mostrando un paquete turístico con características opuestas al utilizado en este caso. Además, con lo anterior, se podría utilizar un olor asociado a un destino invernal para valorar el efecto en comparación al destino veraniego y caribeño de esta investigación. 


\section{DISERTACIONES}

ESTUDIOS

Estudios de audiencias y recepción: audiencias minoritarias y nuevas mediaciones

ISSN: 1856-9536

Doi: http://dx.doi.org/10.12804/revistas.urosario.edu.co/disertaciones/v11i1

Volumen 11, Número 1 / Enero-junio 2018

Versión PDF para imprimir desde

http://revistas.urosario.edu.co/index.php/disertaciones

Considérese que Cox (1967), en una investigación similar a la realizada en el mundo de la moda íntima, concluye que las consumidoras de medias prefieren las aromatizadas con cítrico-naranja antes que las que no cuentan con ningún aroma, ya que consideran de más calidad las que desprenden olor. Con nuestro trabajo, aplicado al caso del sector turismo, se ha visto que mostrando los mismos productos, con y sin estímulo olfativo, no hubo ninguna preferencia por parte de los participantes. En esta línea, resultaría interesante explorar la variable calidad a través del aroma en la intención de compra turística porque, debido a los limitaciones del presente estudio, no se exploró en la prueba experimental, aunque en los antecedentes se vio que el aroma permite evaluar la calidad de un producto o servicio (Chebat \& Michon, 2003).

Cabe señalar una observación no buscada ni consultada bibliográficamente a la que se pudo llegar a través de los resultados. Existe una variable que sí afecta la intención de compra de un destino turístico: el agrado del spot publicitario. Se observó que los sujetos a quienes les gustó el video manifestaron una mayor probabilidad de visitar el destino turístico. Se concluye además que el aroma no influye en el agrado del video, ya que a algunos de los participantes de las dos condiciones experimentales les gustó el video.

También resulta importante la diferenciación hallada en los resultados entre los sujetos dispuestos a pagar por un viaje a un destino turístico y los dispuestos a ir al mismo lugar. Autores como Wahab, Crampón y Rothfield (1976) o Arrebola (1983) mencionaron la importancia de los factores económicos en la decisión de compra de los viajeros. En este estudio, se ha podido confirmar la relevancia del dinero en la intención de comprar un paquete turístico, ya que se vio que los sujetos participantes mostraban más prudencia al evaluar los ítems económicos que los ítems relacionados con la intención de visitar el lugar propuesto. En consecuencia, es diferente sentir atracción e interés por un sitio a verse dispuesto a invertir en visitarlo. Con esto, se deja abierta una línea futura de investigación que podría consistir en realizar el mismo estudio con personas de edades más avanzadas y, consecuentemente, con una situación económica más estable o controlando el poder adquisitivo.

\section{Referencias}

1. Achrol, R. S., \& Kotler, P. (1999). Marketing in the network economy. The Journal of Marketing, 63, 146-163.

2. Aertsen, V. U. (2011). El cine como inductor del turismo. La experiencia turística en Vicky, Cristina, Barcelona. Razón y palabra, 77(2). Recuperado de http://www.razonypalabra.org.mx/varia/77\%204a\%20 parte/54_Aersten_V77.pdf

3. Agapito, D., Valle, P. O. D., \& Mendes, J. (2012). Sensory marketing and tourist experiences. Spatial and Organizational Dynamics Discussions Papers, 10, 7-19.

4. de Almeida, L. G. B. (2015). Cinema, representaçaoes do urbano e identidades: Um olhar sobre or marketing de destinos em Vicky Cristina Barcelona, TURyDES, 8(18).

5. Areni, C. S., \& Kim, D. (1993). The influence of background music on shopping behavior: classical versus top-forty music in a wine store. Advances in Consumer Research, 20(1), 336-340.

6. Arrebola, J. L. S. (1983). La decisión de compra del turista-consumidor. Estudios turísticos, 79, 39-53.

7. Ajzen, I., \& Fishbein, M. (1977). Attitude-behavior relations: A theoretical analysis and review of empirical research. Psychological Bulletin, 84(5), 888-918. 


\section{DISERTACIONES}

ESTUDIOS

Estudios de audiencias y recepción: audiencias minoritarias y nuevas mediaciones

ISSN: 1856-9536

Doi: http://dx.doi.org/10.12804/revistas.urosario.edu.co/disertaciones/v11i1

Volumen 11, Número 1 / Enero-junio 2018

Versión PDF para imprimir desde

http://revistas.urosario.edu.co/index.php/disertaciones

8. Back, R. M. (2015). An investigation into the effect of consumer experience tourism on brand loyalty and purchasing behavior. Doctoral Sissertations May 2014- current. Paper 423. Recueprado de http://scholarworks.umass.edu/dissertations_2/423

9. Baker, J., Grewal, D., \& Parasuraman, A. (1994). The influence of store environment on quality inferences and store image. Journal of the Academy of Marketing Science, 22(4), 328-339.

10. Baker, D. A., \& Crompton, J. L. (2000). Quality, satisfaction and behavioral intentions. Annals of Tourism Research, 27(3), 785-804.

11. Barnett, K. (1972). A survey of the current utilization of touch by health team personnel with hospitalized patients. International Journal of Nursing Studies, 9(4), 195-209.

12. Barreto, J. J., \& Martínez, S. C. (2016). Marketing experiencial en FITUR: análisis de dos destinos competidores, Islas Canarias e Islas Baleares. PAsos Revista de Turismo y Patrimonio Cultural, 14(1). 75-91.

13. Barrios, M. (2012). Marketing de la Experiencia: principales conceptos y características. Palermo Business Review, 7, 67-89.

14. Bedolla Pereda, D. (2002). Diseño sensorial. Las nuevas pautas para la innovación, especialización y personalización del producto. (Tesis doctoral, Universitat Politècnica de Catalunya). Recuperado de http://www. tdx.cat/handle/10803/6826

15. Bellizzi, J. A., \& Hite, R. E. (1992). Environmental color, consumer feelings, and purchase likelihood. Psychology \& Marketing, 9(5), 347-363.

16. Bellos, I., \& Kavadias, S. (2011). The design challenges of experiential services. Recuperado de http:// papers.ssrn.com/sol3/papers.cfm?abstract_id=1912166

17. Bernd H. Schmitt (1999). Experiential marketing. Journal of Marketing Management, 15, 53-67.

18. Bone, P. F., \& Jantrania, S. (1992). Olfaction as a cue for product quality. Marketing Letters, 3(3), 289-296.

19. Boyd Jr, H. W., \& Levy, S. J. (1999). New dimension in consumer analysis. En J. Sidney Levy (Ed), Brands, consumers, symbols and research (p. 474-488). Thousand Oaks: Sage.

20. Braidot, N. (2010). Neuromarketing aplicado. Recuperado de http://s3.amazonaws.com/academia.edu. documents/32307197/506_Neuromarketing_Aplicado.pdf?AWSAccessKeyld=AKIAJ56TQJRTWSMTNPEA\&Expires $=1467560181 \&$ Signature $=$ dlyCATNeN8QenHcNx4KAJeKUtCE\%3D\&response-content-disposition=inline\%3B\%20filename\%3DNeuromarketing_aplicado_Nueva_plataforma.pdf

21. Brakus, J. J., Schmitt, B. H., \& Zarantonello, L. (2009). Brand experience: What is it? How is it measured? Does it affect loyalty? Journal of Marketing, 73(3), 52-68.

22. Cahill, L., Babinsky, R., Markowitsch, H. J., \& McGaugh, J. L. (1995). The amygdala and emotional memory. Nature 377(6547), 295-296.

23. Carù, A., \& Cova, B. (2003). Revisiting consumption experience a more humble but complete view of the concept. Marketing Theory, 3(2), 267-286.

24. Catalunya, U. (2016). Marketing sensorial: comunicación a través de los sentidos. Uoc.edu. Recuperado de http://www.uoc.edu/divulgacio/comein/es/numero38/articles/Article-Elisenda-Estanyol.html

25. Craig-Smith, S. J., Davidson, M., \& French, C. N. (1994). Hospitality and tourism education in Australia: Challenges and opportunities. Ponencia presentada en la Tourism and Research Education in Australia Conference, Canberra. 


\section{DISERTACIONES}

ESTUDIOS

26. Csikszentmihalyi, M., \& Csikszentmihalyi, I. S. (1991). Adventure and the flow experience. En J. C. Miles \& S. Priest (Eds.), Adventure Education (pp. 149-155). State College: Venture.

27. Díez, B. S., Giménez, E. A., \& Delgado, C. F. (2000). Los efectos de las variables ambientales sobre la conducta del consumidor. Distribucion y Consumo, 10(54), 5-24.

28. Digout, J. (2007). Market-in-mind.com. Recuperado de http://www.scribd.com/doc/8529884/Dossier-MarketingexperientielSpecial-on-Experiential-Marketing

29. Dodds, W. B., Monroe, K. B., \& Grewal, D. (1991). Effects of price, brand, and store information on buyers' product evaluations. Journal of Marketing Research, 28, 307-319.

30. Filser, M. (2002). Le marketing de la production d'expérience: statut théorique et implications managériales. Décisions Marketing, 28, 13-22.

31. Filser, M. (2003). Le marketing sensoriel: la quête de l'intégration théorique et managériale. Revue Française du Marketing, 194(4), 5.

32. Fiore, A. M., Yah, X., \& Yoh, E. (2000). Effects of a product display and environmental fragrancing on approach responses and pleasurable experiences. Psychology and Marketing, 17(1), $27-54$.

33. Frias, D. M., Rodriguez, M. A., \& Castañeda, J. A. (2008). Internet vs. travel agencies on pre-visit destination image formation: An information processing view. Tourism Management, 29(1), 163-179.

34. Fuentes, R. C., Moreno-Gil, S., González, C. L., \& Ritchie, J. B. (2015). La creación y promoción de experiencias en un destino turístico. Un análisis de la investigación y necesidades de actuación. Cuadernos de Turismo, 35, 71-94.

35. Garlin, F. V., \& Owen, K. (2006). Setting the tone with the tune: A meta-analytic review of the effects of background music in retail settings. Journal of Business Research, 59(6), 755-764.

36. Golder, P. N., Mitra, D., \& Moorman, C. (2012). What is quality? An integrative framework of processes and states. Journal of Marketing, 76(4), 1-23.

37. Gómez, M., \& García, C. (2012). Marketing sensorial, como desarrollar la atmosfera del establecimiento comercial. Distribución y Consumo, 24, 30-39.

38. Gong, T., \& Tung, V. W. S. (2016). The impact of tourism mini-movies on destination image: The influence of travel motivation and advertising disclosure. Journal of Travel \& Tourism Marketing, 34(3), 416-428.

39. González Gómez, M. C. (2016). Marketing sensorial. Influencia y evolución en el sector hotelero. (Trabajo de fin de grado, Universidad de Valladolid, España). Recuperado de https://uvadoc.uva.es/bitstream/10324/16918/1/TFG-0\%20721.pdf

40. Grewal, D., Monroe, K. B., \& Krishnan, R. (1998). The effects of price-comparison advertising on buyers' perceptions of acquisition value, transaction value, and behavioral intentions. The Journal of Marketing, 46-59.

41. Gulas, C. S., \& Bloch, P. H. (1995). Right under our noses: Ambient scent and consumer responses. Journal of Business and Psychology, 10(1), 87-98.

42. Herrera, J. S. (2012). Nuevas tendencias en comunicación. Madrid: EsIc Editorial.

43. Hirschman, E. C., \& Holbrook, M. B. (1982). Hedonic consumption: Emerging concepts, methods and propositions. The Journal of Marketing, 92-101. 


\section{DISERTACIONES}

ESTUDIOS

Estudios de audiencias y recepción: audiencias minoritarias y nuevas mediaciones

ISSN: 1856-9536

Doi: http://dx.doi.org/10.12804/revistas.urosario.edu.co/disertaciones/v11i1

Volumen 11, Número 1 / Enero-junio 2018

Versión PDF para imprimir desde

http://revistas.urosario.edu.co/index.php/disertaciones

44. Hirschman, E. C., \& Holbrook, M. B. (1992). Postmodern consumer research: The study of consumption as text (Vol. 1). Newbury Park: Sage Publications.

45. Holbrook, M. B., \& Hirschman, E. C. (1982). The experiential aspects of consumption: Consumer fantasies, feelings, and fun. Journal of Consumer Research, 9(2), 132-140.

46. Holbrook, M. B. (1983). Using a structural model of halo effect to assess perceptual distortion due to affective overtones. Journal of Consumer Research, 10(2), 247-252.

47. Howes, D. (2007). Multi-Sensory Marketing in Cross-Cultural Perspective (part I), From Synergy to Synesthesia. Percepnet-Ciencia. Recuperado de http://www.percepnet.com/cien01_07_ang.htm

48. Hudson, S., \& Ritchie, J. R. (2009). Branding a memorable destination experience. The case of 'Brand Canada'. International Journal of Tourism Research, 11(2), 217-228.

49. Hui-Yi, H., \& Hung-Yuan, P. (2010, August). Use behaviors and website experiences of facebook community. En Electronics and Information Engineering (ICEIE), 2010 International Conference On (Vol. 1, pp. V1-379). IEEE.

50. Hultén, B., Broweus, N., \& Van Dijk, M. (2009). What is sensory marketing? Hampshire: Palgrave Macmillan uk.

51. Hultén, B. (2011). Sensory marketing: the multi-sensory brand-experience concept. European Business Review, 23(3), 256-273.

52. Hultén, B. (2012). Sensory cues and shoppers' touching behaviour: The case of IKEA. International Journal of Retail \& Distribution Management, 40(4), 273-289.

53. Igartua, J. J. (2006). Métodos cuantitativos de investigación en comunicación. Barcelona: Bosch.

54. Jiménez Barreto, J., \& Campo Martínez, S. (2016). Experiential marketing in FITUR: analysis of two destinies competitors, Canary and Baleares Islands. PAsos Revista de Turismo y Patrimonio Cultural, 14(1), 75-91.

55. Klatzky, R. L., \& Lederman, S. J. (1992). Stages of manual exploration in haptic object identification. Perception \& Psychophysics, 52(6), 661-670.

56. Koehl, J. L. (2004). Le marketing sensoriel. Recuperado de http://ressources.aunege.fr/nuxeo/site/ esupversions/50a9c6c7-e3a4-4afe-be36-0e9f511f3fe0

57. Konecnik M., (2002). The image as a possible source of competitive advantage of the destination - The case of Slovenia. Tourism Review, 57, 6-12.

58. Kotler, P. (1973). Atmospherics as a marketing tool. Journal of retailing, 49(4), 48-64.

59. Kotler, P. (1999). El marketing según Kotler: cómo crear, ganar y dominar los mercados. Barcelona: Paidós Ibérica.

60. Kreuzer, C. (2006). Emotions and associations to effect pigments. Market research analysis about colors. Darmstadt Merck KGaA, Pigments division.

61. Krishna, A. (Ed.). (2011). Sensory Marketing: research on the sensuality of products. New York: Routledge.

62. Labrecque, L. I., \& Milne, G. R. (2012). Exciting red and competent blue: The importance of color in marketing. Journal of the Academy of Marketing Science, 40(5), 711-727.

63. Lederman, S. J., \& Klatzky, R. L. (1993). Extracting object properties through haptic exploration. Acta Psychologica, 84(1), 29-40.

64. Lenderman, M. (2008). Marketing experiencial: la revolución de las marcas. Madrid: Esıc Editorial.

65. Manzano, R., Serra, T., \& Gavilán, D. (2011). Marketing sensorial: comunicar a través de los sentidos. Harvard-Deusto Marketing \& Ventas, 103, 28-33. 


\section{DISERTACIONES}

ESTUDIOS

Estudios de audiencias y recepción: audiencias minoritarias y nuevas mediaciones

ISSN: 1856-9536

Doi: http://dx.doi.org/10.12804/revistas.urosario.edu.co/disertaciones/v11i1

Volumen 11, Número 1 / Enero-junio 2018

Versión PDF para imprimir desde

http://revistas.urosario.edu.co/index.php/disertaciones

66. Outrebon, M. (2016). Marketing sensorial: el marketing de los 5 sentidos. Espacio Blanco. Recuperado de http://www.espacioblanco.es/los-5-sentidos-aplicados-al-marketing-marketing-sensorial/

67. Maslow, A. H. (1964). Religions, values, and peak-experiences (Vol. 35). Columbus: Ohio State University Press.

68. Medlik, S., \& Middleton, V. T. (1973). Product formulation in tourism. Tourism and Marketing, 13, 173-201.

69. Michon, R., Chebat, J. C., \& Turley, L. W. (2005). Mall atmospherics: The interaction effects of the mall environment on shopping behavior. Journal of Business Research, 58(5), 576-583.

70. Milliman, R. E. (1982). Using background music to affect the behavior of supermarket shoppers. The Journal of Marketing, 46(3), 86-91.

71. Milliman, R. E. (1986). The influence of background music on the behavior of restaurant patrons. Journal of Consumer Research, 13(2), 286-289.

72. Mitchell, D. J., Kahn, B. E., \& Knasko, S. C. (1995). There's something in the air: Effects of congruent or incongruent ambient odor on consumer decision making. Journal of Consumer Research, 22(2), 229-238.

73. Moral, M. M., \& Alles, M. T. F. (2012). Nuevas tendencias del marketing: el marketing experiencial. Entelequia: Revista Interdisciplinar, 14, 237-251.

74. Morrin, M., \& Ratneshwar, S. (2000). The impact of ambient scent on evaluation, attention, and memory for familiar and unfamiliar brands. Journal of Business Research, 49(2), 157-165.

75. Morton, J.L. (1995). Color matters. Recuperado de http://www.colormatters.com/

76. Navalles, P. (2011). Los olores como marca comercial. Revista de Rinologia, 11, 23-28.

77. Oh, H., Fiore, A. M., \& Jeoung, M. (2007). Measuring experience economy concepts: Tourism applications. Journal of Travel Research, 46(2), 119-132.

78. Olahut, R., M., Plaias I. (2013). The effects of ambient scent on consumer behavior: A review of the literature. Annals of Faculty of Economics, 1(1), 1797-1806.

79. Oliver, C. (1997). Sustainable competitive advantage: Combining institutional and resource-based views. Strategic Management Journal, 18(9), 697-713.

80. Patterson, A., Hodgson, J., \& Shi, J. (2008). Chronicles of 'customer experience': The downfall of Lewis's foretold. Journal of Marketing Management, 24(1-2), 29-45.

81. Peck, J., \& Childers, T. L. (2003). To have and to hold: The influence of haptic information on product judgments. Journal of Marketing, 67(2), 35-48.

82. Peck, J., \& Wiggins, J. (2006). It just feels good: Customers' affective response to touch and its influence on persuasion. Journal of Marketing, 70(4), 56-69.

83. Pine, B. J., \& Gilmore, J. H. (1998). Welcome to the experience economy. Harvard business review, 76, 97-105.

84. Pine, B. J., \& Gilmore, J. H. (1999). The experience economy: work is theatre \& every business a stage. Boston: Harvard Business Press.

85. Prayag, G. (2007). Assessing international tourists' perceptions of service quality at Air Mauritius. International Journal of Quality \& Reliability Management, 24(5), 492-514.

86. Prayag, G. (2009). Tourist evaluations of destination image, satisfaction, and future behavioral intentions. The case of Mauritius. Journal of Travel \& Tourism Marketing, 26(8), 836-853. 


\section{DISERTACIONES}

ESTUDIOS

87. Raisamo, R., Patomäki, S., Hasu, M., \& Pasto, V. (2007). Design and evaluation of a tactile memory game for visually impaired children. Interacting with Computers, 19(2), 196-205.

88. Rajotte, M. (2010). Le Marketing Sensoriel. Quebec: Bibliothèque Nationale du Québec. Recuperado de https://www.economie.gouv.qc.ca/fileadmin/contenu/publications/outils_aide/gestion_entreprise/ DDE_sensoriel.pdf

89. Rieunier, S. (2000). L'influence de la musique d'ambiance sur le comportement des consommateurs sur le lieu de vente. (Tesis doctoral. Université Paris IX-Dauphine, Francia).

90. Ritchie J.R., B., Tung V.W., S., \& Ritchie R.J., B. (2010). Tourism experience management research Emergence, evolution and future directions. International Journal of Contemporary Hospitality Management, 23(4), 419-438.

91. Romera Ferrera, J. (2014). Marketing sensorial en el punto de venta: el caso Hollister. (Tesis de grado. Universitat Autònoma de Barcelona, España).

92. Ryan, C., \& Glendon, I. (1998). Application of leisure motivation scale to tourism. Annals of Tourism Research, 25(1), 169-184.

93. Schmitt, B. (2000). Experiential Marketing. Bilbao: Ediciones Deusto.

94. Schmitt, B. H. (2010). Customer experience management: A revolutionary approach to connecting with your customers. Hoboken: John Wiley \& Sons.

95. Schmitt, B., \& Simonson, A. (1998). Marketing y estética: la gestión estratégica de la marca, la identidad y la imagen. Bilbao: Ediciones Deusto.

96. Shaw, C., \& Ivens, J. (2005). Building great customer experiences. Basingstoke: Palgrave Macmillan.

97. Simonson, A., \& Schmitt, B. H. (1997). Marketing Aesthetics: The Strategic Management of Brands, Identity, and Image. Simon and Schuster.

98. Singh, S. (2006). Impact of color on marketing. Management Decision, 44(6), 783-789.

99. Smith, W. L. (2005). Experiential tourism around the world and at home: definitions and standards. International Journal of Services and Standards, 2(1), 1-14.

100.Sotomayor, M. I., \& del Perú, O. T. K. La experiencia turística de los sentidos. Cultura, 26, 151-178. Recuperado se http://www.revistacultura.com.pe/revistas/RCU_26_1_la-experiencia-turistica-de-los-sentidos. pdf

101.Spangenberg, E. R., Crowley, A. E., \& Henderson, P. W. (1996). Improving the store environment: do olfactory cues affect evaluations and behaviors? The Journal of Marketing, 60(2), 67-80.

102.Srinivasan, S. R., \& Srivastava, R. K. (2010). Creating the futuristic retail experience through experiential marketing: Is it possible\&quest; An exploratory study. Journal of Retail \& Leisure Property, 9(3), 193-199.

103. Sweeney, J. C., \& Wyber, F. (2002). The role of cognitions and emotions in the music-approach-avoidance behavior relationship. Journal of services marketing, 16(1), 51-69.

104. Thorne, F. C. (1963). The clinical use of peak and nadir experience reports. Journal of Clinical Psychology, 19(2), 248-250.

105. Tiu Wright, L., Newman, A., \& Dennis, C. (2006). Enhancing consumer empowerment. European Journal of Marketing, 40(9/10), 925-935. 


\section{DISERTACIONES}

106. Tribe, J., \& Snaith, T. (1998). From SERVQUal to Holsat: Holiday satisfaction in Varadero, Cuba. Tourism Management, 19(1), 25-34.

107. Tsaur, S. H., Chiu, Y. T., \& Wang, C. H. (2007). The visitors behavioral consequences of experiential marketing: An empirical study on Taipei Zoo. Journal of Travel \& Tourism Marketing, 21(1), 47-64.

108. Uriely, N. (2005). The tourist experience: Conceptual developments. Annals of Tourism research, 32(1), 199-216.

109.Verhoef, P. C., Lemon, K. N., Parasuraman, A., Roggeveen, A., Tsiros, M., \& Schlesinger, L. A. (2009). Customer experience creation: Determinants, dynamics and management strategies. Journal of retailing, 85(1), 31-41.

110.Vernet, F., \& Rieunier, S. (2004). Le marketing sensoriel chez Nature \& Découvertes: 10 ans d'évolution de l'offre sensorielle en restant à l'écoute du consommateur. Décisions Marketing, 33, 77-80.

111.Wahab, S., Crampon, L. J., \& Rothfield, L. M. (1976). Tourism marketing: a destination-orientated programme for the marketing of international tourism. Londres: Tourism International Press.

112. Walls, A. R., Okumus, F., Wang, Y. R., \& Kwun, D. J. W. (2011). An epistemological view of consumer experiences. International Journal of Hospitality Management, 30(1), 10-21.

113.Wang, D., Xiang, Z., \& Fesenmaier, D. R. (2016). Smartphone use in everyday life and travel. Journal of Travel Research, 55(1), 52-63.

114.Wang, N. (1999). Rethinking authenticity in tourism experience. Annals of tourism research, 26(2), 349-370.

115. Ward, P., Davies, B. J., \& Kooijman, D. (2003). Ambient Smell and the Retail Environment: Relating Olfaction Research to Consumer Behavior. Journal of Business and Management, 9(3), 289.

116.Wright, L.T., Newman, A. y Dennis, C. (2006). Enhancing consumer empowerment, European Journal of Marketing, 40(9/10), 925-935.

117. Yalch, R., \& Spangenberg, E. (1990). Effects of store music on shopping behavior. Journal of Consumer Marketing, 7(2), 55-63.

118. Yalch, R. F., \& Spangenberg, E. R. (2000). The effects of music in a retail setting on real and perceived shopping times. Journal of Business Research, 49(2), 139-147.

119.Zarantonello, L., \& Schmitt, B. H. (2013). The impact of event marketing on brand equity: The mediating roles of brand experience and brand attitude. International Journal of Advertising, 32(2), 255-280.

120.Zheng, L. (2006). Research on the Experiential Tourism of Rural Area in Eastern of Liaoning Province. Ecological Economy, 6, 029. 